\title{
Fabricating PC/PAN composite membranes by vapor-induced phase separation
}

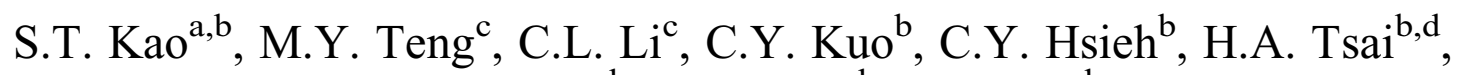 \\ D.M. Wang ${ }^{\mathrm{b}, \mathrm{e}}$, K.R. Lee ${ }^{\mathrm{b}}$, J.Y. Lai ${ }^{\mathrm{b}}$ \\ ${ }^{a}$ Institute of Polymer Science and Engineering, National Taiwan University, Taipei 10617, Taiwan \\ ${ }^{b} R \& D$ Center for Membrane Technology and Department of Chemical Engineering, \\ Chung Yuan University, Chungli 32023, Taiwan \\ Tel. +886-3 2654190; Fax +886-3 2654198; email: krlee@cycu.edu.tw \\ ${ }^{c}$ Department of Chemical and Material Engineering, Nanya Institute of Technology, \\ Chungli 32034, Taiwan \\ ${ }^{d}$ Department of Material and Fiber, Nanya Institute of Technology, Chungli 32034, Taiwan \\ ${ }^{e}$ Department of Chemical Engineering, National Taiwan University, Taipei 10617, Taiwan
}

Received 15 August 2007; accepted revised 25 September 2007

\begin{abstract}
In this study, a series of polycarbonate/polyacrylonitrile (PC/PAN) composite membrane has been successfully fabricated by vapor-induced phase separation and in-situ casting process. The effect of the casting solution concentration on the membrane morphology was investigated. The morphology of the PC/PAN composite membranes was characterized by SEM. The effect of the skin layer thickness on the water flux was measured at a fixed pressure. Compared with the traditional asymmetric PAN membrane with a dense skin layer and porous support layer, the PC/PAN composite membranes prepared by in-situ casting process effectively prevent the skin layer formation, resulting in the water flux increases.
\end{abstract}

Keywords: Vapor-induced phase separation; In-situ casting process; Composite membranes; Skin-free

\section{Introduction}

Asymmetric polymeric membranes, with dense skin layer and porous sublayer have been

*Corresponding author.

Presented at the Fourth Conference of Aseanian Membrane Society (AMS 4), 16-18 August 2007, Taipei, Taiwan. 
and/or a nonsolvent (dry process); (2) exposure to a nonsolvent vapor, such as water vapor, which would be absorbed on the exposed surface (VIPS); (3) immersing a casting solution in a nonsolvent bath (NIPS); (4) thermal induced phase separation (TIPS) $[1,2]$. Those processes are common methods to product asymmetric polymeric membranes. Nonsolvent induced phase separation is widely adopted and studied. A typical asymmetric membrane, using nonsolvent induced phase separation to product, consists of a dense skin layer and a porous sublayer. The asymmetric membranes with dense skin layer often lead to low permeability coefficients. Thus, in order to improve the disadvantage of low permeation rate, membranes with asymmetric morphology and thin dense skin layer are more suitable. Therefore, understanding the formation mechanism of asymmetric membranes, which is helpful in controlling the membrane morphology, is of great importance.

In this study, the membrane formation was combined the vapor-induced phase separation (VIPS) process and in-situ casting process [3-8]. Firstly, the cast film was exposed to the atmosphere a period of time, called VIPS time. And then, the film was immersed into the nonsolvent bath. This method can effectively suppress the formation of macrovoids and obtain porous top surface at the same time. The in-situ casting method allows to using different polymer solutions to form each layer of the ultimate membrane at the same time. Through the polymer pair selection and/or composition arrangement, a skin-free PC/PAN composite membrane can be prepared by VIPS and in-situ casting process.

\section{Experimental}

\subsection{Material}

Polycarbonate (Uplion-2000) was supplied by Mitsubishi Gas Chemical Co. Polyacrylonitrile (PAN) was supplied by the Tong-Hua synthesis fiber Co. Ltd. (Taiwan). N-methyl-2-pyrrolidone
(NMP) supplied by Aldrich Co. Ltd., was used as the solvent.

\subsection{Membrane preparation}

PC/PAN composite membranes were obtained by in-situ casting two polymer solutions on a glass plate with 300 and $500 \mu \mathrm{m}$ thickness under controlled temperature and relative humidity. The polymer solutions used for obtaining the support and the upper layers were referred to as the solution A (PAN/NMP = 15 wt.\%) and solution $\mathrm{B}$ (PC/NMP $=5-15$ wt.\%), respectively [6-8]. Water vapor was absorbed from the ambient air and phase separation occurred gradually. Fig. 1 shows the schematic representation of the in-situ casting process.

\subsection{SEM observation}

The morphology of PC/PAN composite membranes was observed with the scanning electron microscopy (Hitachi, Model: S-3000N).

\subsection{Pure water flux}

The pure water flux was measured by a deadend filtration setup. It consists of an air cylinder

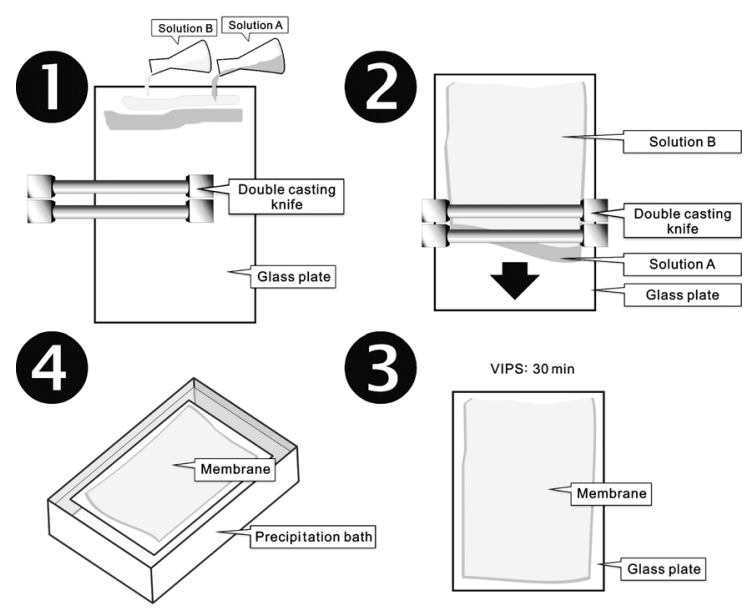

Fig. 1. Schematic diagram of fabricating skin-free membranes using in-situ double casting method. 
to provide constant trans-membrane pressure (TMP), a 5 L stainless steel reservoir, a beaker or a $5 \mathrm{~L}$ plastic pail used as an effluent receiver and an electronic balance to measure the amount of effluent. A testing membrane was placed in stainless steel permeation cell with an inner diameter of $3.3 \mathrm{~cm}$ and an estimated surface area of $8.45 \mathrm{~cm}^{2}$. A sinter metal plate provides support for the membranes. The water flux was measured at $1 \mathrm{~kg} / \mathrm{cm}^{2}$ pressure at room temperature.

\subsection{Mechanical properties measurement}

Tensile stress at break of the composite membranes was measured by using tensile test machine (ASTM D412) at a crosshead speed of $0.5 \mathrm{~mm} / \mathrm{min}$, with a clamp distance of $2.5 \mathrm{~cm}$. The initial Young's modulus was calculated in the range of $0.5-1.0 \%$ tensile strain.

\subsection{Determination of the mean pore diameter and pore size distribution}

In order to define the pore size of the membrane, a capillary flow porometer (CFP-1500-AXEL, PMI) was used. At first, the membrane was wetted by Porewick (surface tension: 16 dynes $/ \mathrm{cm}$ ). Then pressure of a non-reacting gas $\left(\mathrm{N}_{2}\right)$ on the membrane was increased to remove the wetting liquid from pores and permitted gas flow. The bubble point data were used to evaluate the pore size of the membrane. The pore size range was suitable about $0.013-500 \mu \mathrm{m}$ for this method.

\section{Results and discussion}

Permeation rate and separation factors are two important characteristics determining the separation performance of membranes. Thus, to improve the permeation rate of membranes are structurally asymmetric, with a thin skin supported by a porous sublayer.

The most common processes are based on phase inversion of a casting solution. Nonsolvent induced phase separation (NIPS) is widely adopted and studied. An asymmetric PAN membrane with a dense skin layer and a porous sublayer was prepared via a nonsolvent induced phase separation process, as shown in Fig. 2A1 and A2.

The disadvantage of using the above membranes for separation is the low permeation rate. How to overcome this advantage? Vapor-induced phase separation (VIPS) was investigated in this article, In general, the VIPS method can effectively suppress the formation of macrovoids and obtain porous top surface at the same time. However, very similar membrane morphology was observed (Fig. 2B1 and B2) from the membrane prepared by VIPS method. These results indicated that the asymmetric PAN membrane with porous top skin can not be obtained by using VIPS method. However, if we want to use the asymmetric PAN membrane for the membrane separation processes, how to prepare a skin-free PAN membrane to reduce the mass transport resistance is a very important issue. From the membrane formation point of view, polycarbonate (PC) can be alternative to the PAN because of its good chemical stability, strong mechanical

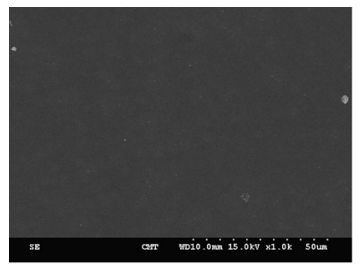

(A1) Surface $\times 1 \mathrm{k}$

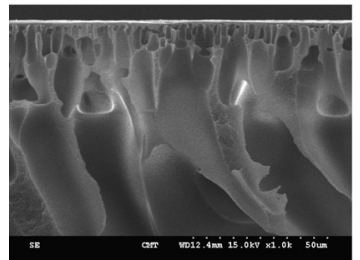

(A2) Cross-section $\times 1 \mathrm{k}$

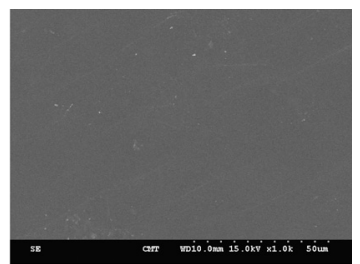

(B1) Surface $\times 1 \mathrm{k}$

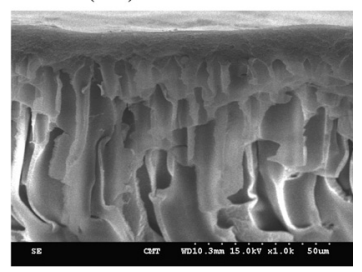

(B2) Cross-section $\times 1 \mathrm{k}$
Fig. 2. Membrane structures obtained by the regular casting of $15 \mathrm{wt} \%$ PAN/NMP solution: (A) NIPS; (B) VIPS (exposed time: $30 \mathrm{~min}$; relative humidity: $70 \%$; temperature: $25^{\circ} \mathrm{C}$ ). 


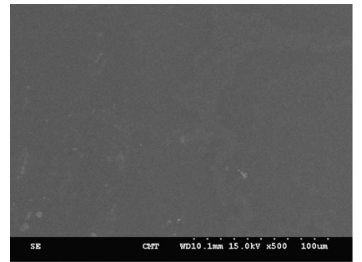

(A1) Surface $\times 1 \mathrm{k}$

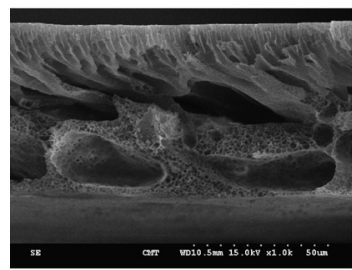

(A2) Cross-section $\times 1 \mathrm{k}$
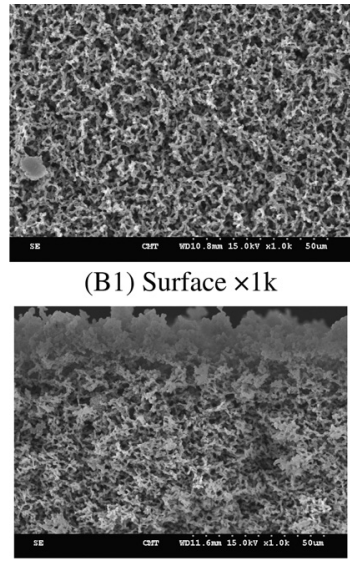

(B1) Surface $\times 1 \mathrm{k}$

(B2) Cross-section $\times 1 \mathrm{k}$

Fig. 3. Membrane structures obtained by the regular casting of 15 wt.\% PC/NMP solution: (A) NIPS; (B) VIPS (exposed time: $30 \mathrm{~min}$; relative humidity: 70\%; temperature: $25^{\circ} \mathrm{C}$ ).

strength and membrane formation properties. Fig. 3A1 and A2 shows that the asymmetric PC membrane prepared by using NIPS method. Compared with the asymmetric PAN membrane (Fig. 2A1 and A2), a similar membrane morphology of asymmetric PC membrane was obtained. The membrane structure still consists of a thin, dense skin layer and a porous sublayer. However, quite different membrane morphology was observed (Fig. 3B1 and B2) from the membrane prepared by VIPS method. These results indicated that the asymmetric PC membrane with porous top skin can be obtained via a VIPS method. The bicontinuous structures were observed, as indicated in Fig. 3B1 and B2.

These results can be explained by the affinity between water vapor and the polymer. The solubility parameter differences between polymer and water is shown in Table 1. It shows that the solubility parameter differences between PAN and water $\left(\Delta \delta=18.5 \mathrm{MPa}^{1 / 2}\right)$ has smaller value. This result shows the water may be a weak nonsolvent for PAN, therefore the VIPS method cannot fabricate an asymmetric PAN membrane with porous top surface. The weak
Table 1

Solubility parameter differences between polymer and water

\begin{tabular}{lll}
\hline & $\delta_{\mathrm{t}}\left(\mathrm{MPa}^{1 / 2}\right)$ & $\Delta \delta_{\mathrm{t}}\left(\mathrm{MPa}^{1 / 2}\right)$ \\
\hline PAN & 29.4 & 18.5 \\
PC & 19.7 & 28.2 \\
Water & 47.9 & - \\
\hline
\end{tabular}

nonsolvent had the higher affinity between water vapor and the PAN membrane, resulting in the PAN membrane still with a dense skin layer.

Compared with the solubility parameter difference value of PAN formation system, the PC membrane formation system has the higher value $\left(\Delta \delta=28.2 \mathrm{MPa}^{1 / 2}\right)$. The skin-free PC membrane may be resulted in the strong nonsolvent effect. During the VIPS stage, the water vapor can be drawn into the casting solution, initiating solvent migration and redistribution of polymer in the casting film.

Because aforesaid phenomenon adds strong nonsolvent effect causes microphase separation, resulting in the PC membrane with a porous top layer. In addition, the viscosity of PC casting solution system is much lower than that of the PAN casting solution system, resulting in the diffusion coefficient of water and NMP in the former system are higher than that of the latter system. The polymer concentration at the interface between the PC casting solution and the water vapor decreases rapidly. Due to these two reasons (solubility parameter differences and polymer solution viscosity), a quite different PC membrane morphology was observed in this article. Moreover, from the view point of cloud point, defined as the volume of coagulant required bringing the casting solution into liquid-liquid demixing, can be used to evaluate how easy the casting solution is to demix. The system with low nonsolvent content during the NIPS process, indicating the casting solution easier to phase separate, corresponds to higher membrane 
Table 2

The cloud point and viscosity of the polymer solution systems $^{\mathrm{a}}$

\begin{tabular}{lll}
\hline $\begin{array}{l}\text { Polymer } \\
\text { solution }\end{array}$ & $\begin{array}{l}\text { Viscosity } \\
(\mathrm{cp})\end{array}$ & $\begin{array}{l}\text { Cloud point } \\
\mathrm{H}_{2} \mathrm{O}(\text { vol.\%) }\end{array}$ \\
\hline PAN/NMP & 9120 & 8.87 \\
PC/NMP & 85 & 3.23 \\
\hline
\end{tabular}

${ }^{\text {a}}$ Polymer solution concentration: $15 \mathrm{wt} . \%$; testing temperature: $25^{\circ} \mathrm{C}$.

porosity. The cloud point of the $15 \mathrm{wt} . \%$ polymer solution systems was shown in Table 2.

The experimentally determined binodal curves for these systems are shown in Fig. 4. It shows that the bimodal curve of PAN casting solution system was located far from the solvent-polymer axis than that of the PC casting solution system. For example, the water content of cloud point at 15 wt.\% PC casting solution system is much lower than that of the PAN casting solution system (Table 2). It means that the demixing area of the former is larger than that of the latter, thus a high porosity membrane surface is obtained.

The asymmetric membranes with dense skin offered mechanical properties, but leads to low permeability coefficients. Table 3 shows the

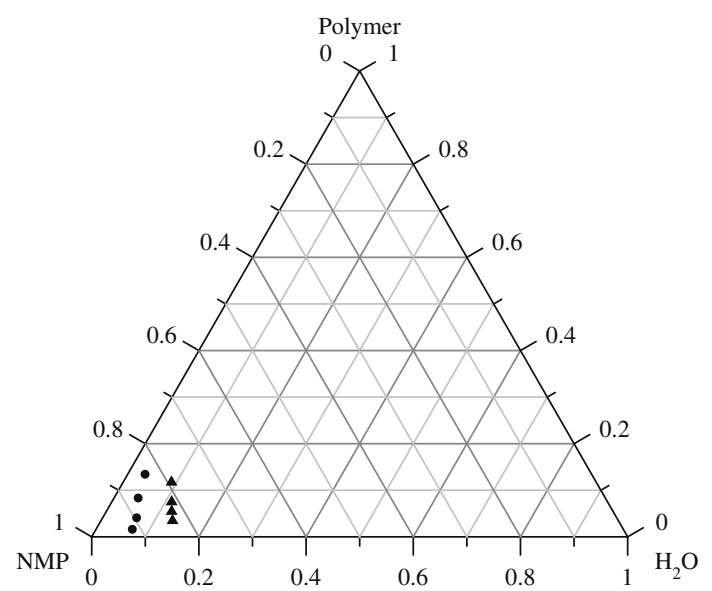

Fig. 4. Ternary phase diagram of polymer/solvent/ coagulant at $25^{\circ} \mathrm{C}$, (○) $\mathrm{PC}(\boldsymbol{\Delta}) \mathrm{PAN}$.
Table 3

Membrane properties for PAN or PC VIPS membranes

\begin{tabular}{lll}
\hline Membrane & $\begin{array}{l}\text { Mechanical } \\
\text { property }\end{array}$ & $\begin{array}{l}\text { Surface } \\
\text { pore }\end{array}$ \\
\hline PAN & $\mathrm{O}$ & $\mathrm{X}$ \\
PC & $\mathrm{X}$ & $\mathrm{O}$ \\
\hline
\end{tabular}

O: good; X: bad.

mechanical properties of the PAN and PC membranes prepared by VIPS method.

It shows that the mechanical properties of the PC membrane with porous top surface are lower than that of the PAN membrane with dense top layer. For polymeric materials, mechanical behavior involves the deformation of a material under the influence of an applied force. In the membrane separation process, the membrane is the key-point which can be considered as a permselective barrier between two phases. Transport through the membrane will take place as a result of the driving force acting on the individual components in the feed. Substances transport across the membrane either by pressure or by concentration difference. To obtain a higher flux, excess pressure will frequently apply to the upstream of the membrane. Consequently, the mechanical properties of the membrane play important roles in prolonged operations. Thus, we try to combine the individual advantage of PC and PAN to prepare a skin-free PC/PAN composite membrane via a VIPS and in-situ casting process.

From Fig. 2A1 and A2, an asymmetric PAN membrane with thick dense skin layer prepared by nonsolvent induce phase separation was observed. The disadvantage of using the above membranes for separation is the low permeation rate. How to overcome this advantage? The in-situ casting process associate with VIPS is one of the candidates of membrane prepared methods.

The SEM micrographs of the PC/PAN composite membrane prepared by in-situ casting process associate with VIPS are shown in Fig. 5. 


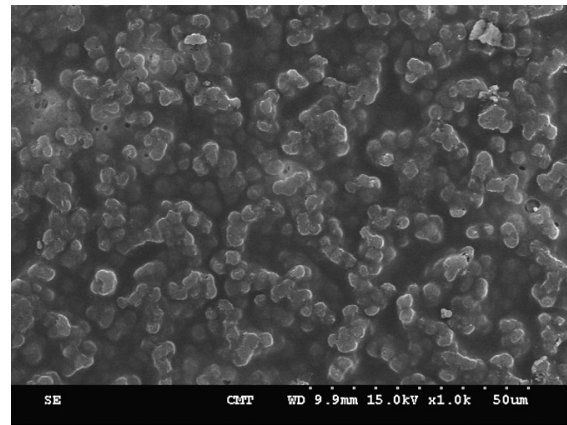

(A) $15 \mathrm{wt} \%$

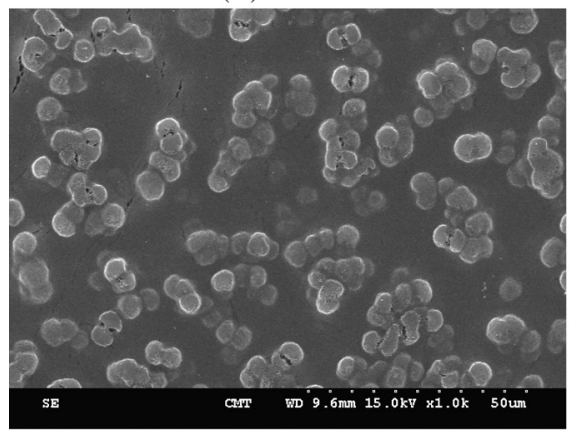

(B) $12 \mathrm{wt} \%$

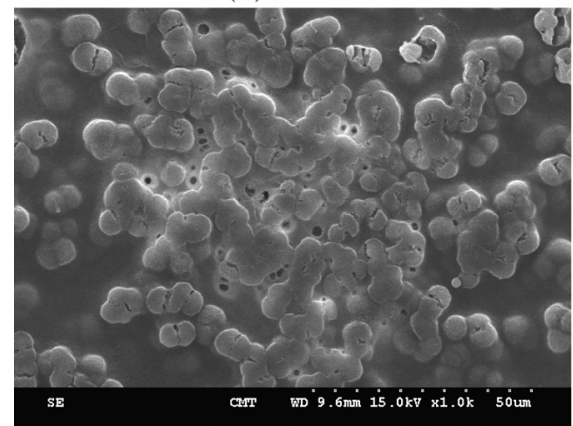

(C) $7 \mathrm{wt} \%$

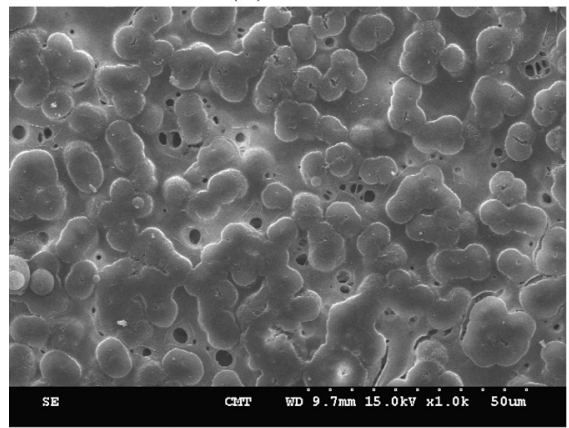

(D) $5 \mathrm{wt} \%$

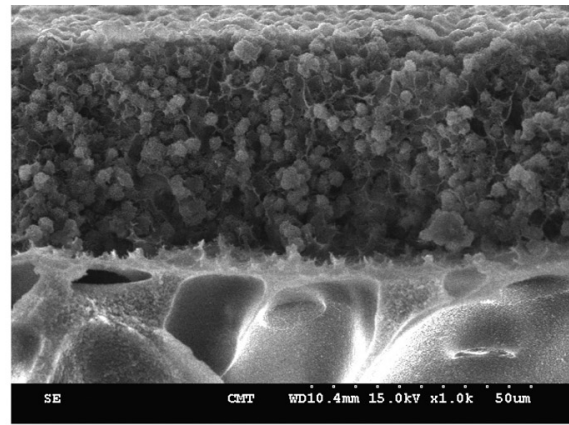

(a) $15 \mathrm{wt} \%$

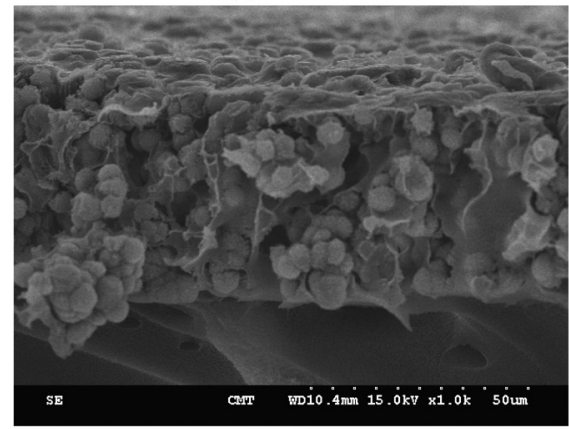

(b) $12 \mathrm{wt} \%$

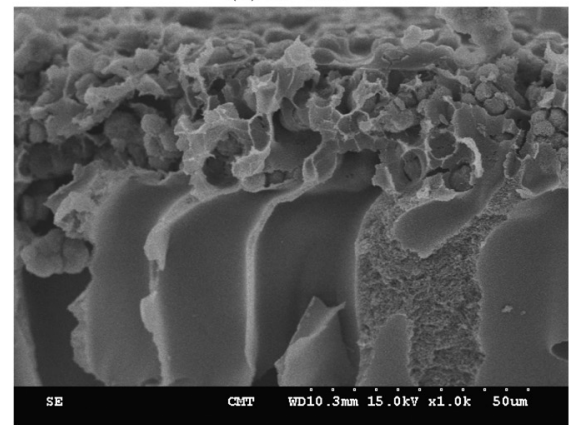

(c) $7 \mathrm{wt} \%$

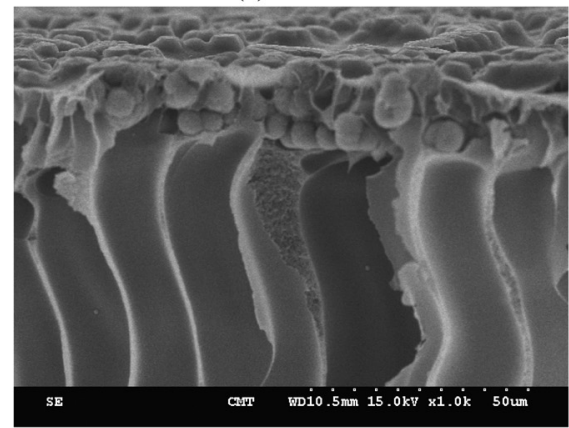

(d) $5 \mathrm{wt} \%$

Fig. 5. (A-D) Surface and (a-d) cross-section morphologies of the membranes obtained by simultaneous casting of 15 wt.\% PAN/NMP and different PC/NMP concentration by VIPS process (exposed time: $30 \mathrm{~min}$; relative humidity: $70 \%$; temperature: $25^{\circ} \mathrm{C}$ ). 
From Fig. 5a-d, it can be found that membranes divided into nodular aggregated and macrovoids layers. But the dense skin layer vanishes. The nodular aggregated layer thickness increases with increasing the PC/NMP concentration. These phenomena might be due to the fact that the viscosity of polymer solution increases with the polymer content increases and the nodular coarsen gradually, resulting in the nodular aggregated layer thickness increases and a skin-free PC/PAN composite membrane can be obtained.

Fig. 6 shows the effect of membrane morphology on the water flux through the PC/PAN composite membrane. It shows that the water flux decreases with the PC concentration increases. These phenomena might be due to the fact that the thickness of the nodular aggregated layer of the PC/PAN composite membrane increases with increasing the top PC layer concentration during the in-situ casting process. These results corresponding to the SEM morphology analysis, as indicated in Fig. 5. In addition, compared with membrane prepared by single layer casting process (PAN), the PC/PAN composite membrane prepared by VIPS and in-situ casting process has

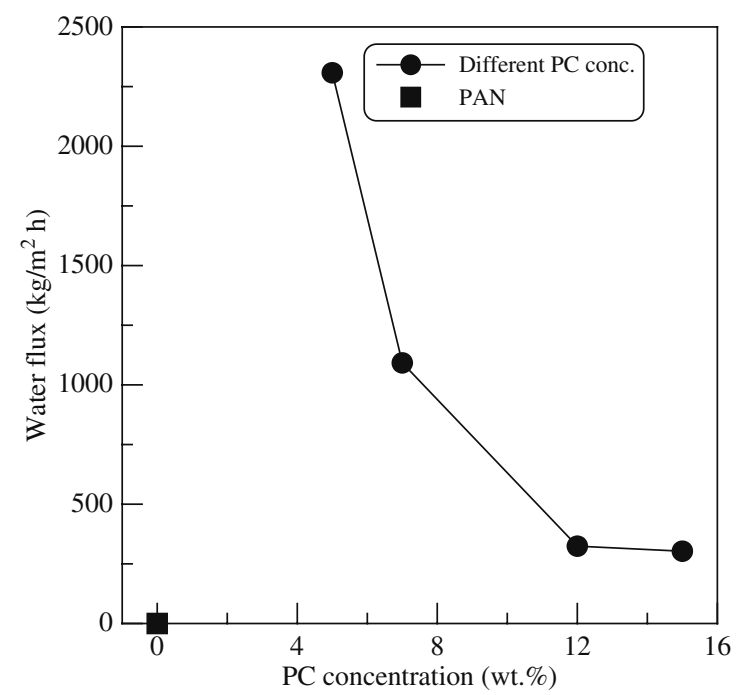

Fig. 6. Effect of PC concentration on the water flux of the PC/PAN composite membrane. the higher water flux. Because of the VIPS and in-situ casting process effectively suppresses the formation of macrovoids and obtain a skin-free membrane structure at the same time.

In this study, the in-situ casting method allows to using different polymer solutions to form each layer of the ultimate membrane at the same time. Four different PC concentrations were investigated about the relationship with the pore size of the surface. Fig. 7 shows the effect of PC concentration on the pore size of the PC/PAN composite membrane. It shows that the pore size decreases with increasing PC concentration. These results corresponding to the SEM morphology analysis very well (Fig. 5). As the polymer concentration increased, the viscosity of casting solution enhanced and therefore the mobility of the polymer chains will be diminished. As a result, a higher resistance is against the coarsening of the polymer poor phase, therefore the nodular size decreases with increasing the PC concentration. The pore size distribution measured with PMI also conformed to SEM observation and water flux analysis. In addition, compared with the PC/PAN composite membranes, the pure PAN membrane prepared by VIPS has low mean

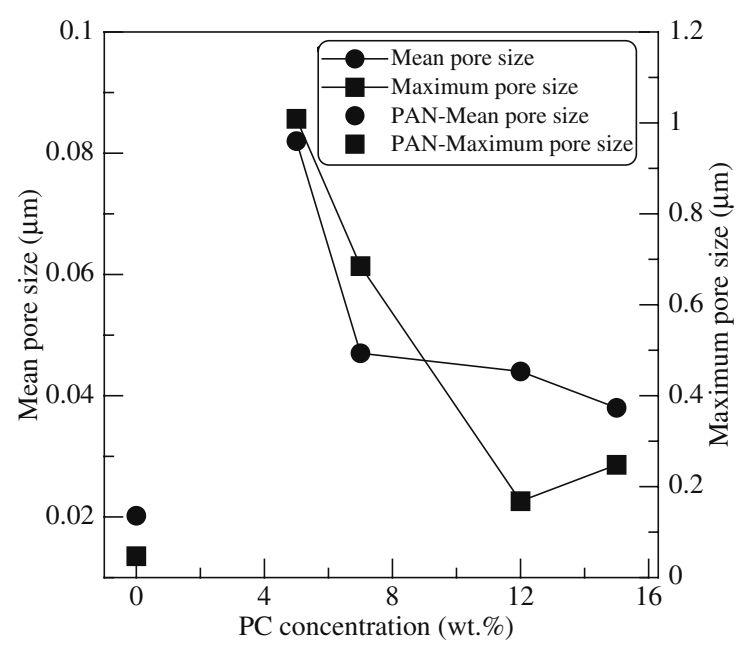

Fig. 7. Effect of PC concentration on the pore size of the PC/PAN composite membrane. 


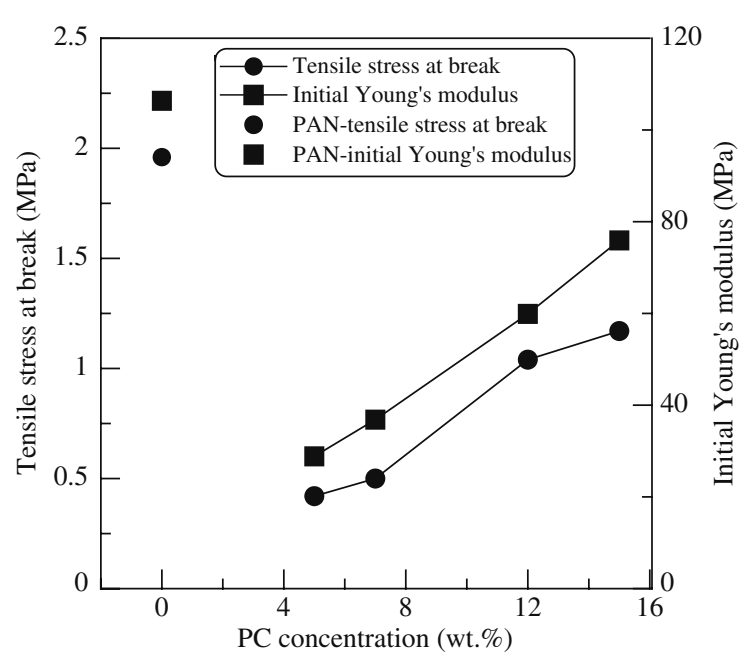

Fig. 8. Effect of PC concentration on the mechanical properties of the PC/PAN composite membrane.

pore size. These results might be because of the skin layer formation during the phase inversion process of the PAN casting solution.

Fig. 8 shows the effect of membrane morphology on the mechanical properties of the PC/PAN composite membrane. All of the mechanical properties (the tensile strength at break, and the initial Young's modulus) increase with increasing the PC concentration. In other words, the mechanical intensity is offered by the nodular aggregated layer thickness. The nodular aggregated layer thickness contributes to the endurance to the applied force and resulted in an increase in the tensile strength at break. However, compared with the skin-free asymmetric PC/PAN composite membrane, the pure PAN membrane has the highest mechanical properties. These phenomena might be due to the dense skin layer effect.

\section{Conclusion}

In this study, a skin-free asymmetric PC/PAN composite membrane was successfully prepared by VIPS and in-situ casting process. From the SEM morphology and analysis of water flux, the PC/PAN composite membranes have low resistance for the permeating species transport. The pore size measured with PMI also conforms to SEM observation and water flux analysis. The mechanical properties of the PC/PAN composite membrane increase with increasing the PC casting layer concentration.

\section{References}

[1] S.J. Shin, J.P. Kim, H.J. Kim, J.H. Jeon and B.R. Min, Preparation and characterization of polyethersulfone microfiltration membranes by a 2-methoxyethanol additive, Desalination, 186 (2005) 1-10.

[2] M.H.V. Mulder, Basic Principles of Membrane Technology, Kluwer Academic Publishers, The Netherlands, 1996.

[3] H. Caquineau, P. Menut, A. Deratani and C. Dupuy, Influence of the relative humidity on film formation by vapor induced phase separation, Polym. Eng. Sci., 43 (2003) 789-808.

[4] H. Matsuyama, M. Teramoto, R. Nakatani and T. Maki, Membrane formation via phase separation induced by penetration of nonsolvent from vapor phase. II. Membrane morphology, J. Appl. Polym. Sci., 74 (1999) 171-178.

[5] H.C. Park, Y.P. Kim, H.Y. Kim and Y.S. Kang, Membrane formation by water vapor induced phase inversion, J. Membr. Sci., 156 (1999) 169-178.

[6] A.J. Reuvers, Membrane formation. Diffusion induced demixing processes in ternary systems, Ph.D. Thesis, University of Twente, The Netherlands, 1987.

[7] C.C. Pereira, R. Noberega and C. P. Borges, Membranes obtained by simultaneous casting of two polymer solutions, J. Membr. Sci., 192 (2001) $11-26$.

[8] C.C. Pereira, R. Nobrega, K.V. Peinemann and C.P. Borges, Hollow fiber membranes obtained by simultaneous spinning of two polymer solutions: a morphological study, J. Membr. Sci., 226 (2003) $35-50$. 